\title{
Revisão: Implantação das boas práticas de fabricação na indústria Brasileira de alimentos
}

\author{
Review: Implementation of good manufacturing practices in the Brazilian food industry \\ Revisión: Implementación de buenas prácticas de fabricación en la industria alimentaria Brasileña
}

Recebido: 05/01/2021 | Revisado: 12/01/2021 | Aceito: 15/01/2021 | Publicado: 18/01/2021

Patricia Ocampos de Oliveira

ORCID: https://orcid.org/0000-0002-3387-1959

Universidade Estácio de Sá, Brasil

E-mail: po2camaraino@hotmail.com

Roberta da Silveira

ORCID: https://orcid.org/0000-0002-0037-4307

Universidade Estadual de Maringá, Brasil

E-mail: dasilveira.roberta@gmail.com

Eloize Silva Alves

ORCID: https://orcid.org/0000-0002-3340-8374

Universidade Estadual de Maringá, Brasil

E-mail: eloizeetaus@ gmail.com

Bruno Henrique Figueiredo Saqueti

ORCID: https://orcid.org/0000-0002-1118-4605

Universidade Estadual de Maringá, Brasil

E-mail: bruno_saqueti@outlook.com

Matheus Campos de Castro

ORCID: https://orcid.org/0000-0002-9918-1491

Universidade Estadual de Maringá, Brasil

E-mail: 1996mcastro@gmail.com

Patricia Magalhães de Souza

ORCID: https://orcid.org/0000-0001-5916-0744

Universidade Estadual de Maringá, Brasil

E-mail: patricia.magalhaes11@hotmail.com

Isadora Boaventura Ponhozi

ORCID: https://orcid.org/0000-0001-7230-161X

Universidade Estadual de Maringá, Brasil

E-mail: isa.ponhozi@gmail.com

Joice Camila Martins da Costa

ORCID: https://orcid.org/0000-0003-2175-7494

Universidade Estadual de Maringá, Brasil

E-mail: joicecamilamart@gmail.com

Janaina Schueler

ORCID: https://orcid.org/0000-0001-9841-6757

Universidade Tecnológica Federal do Paraná, Brasil

E-mail: jana_schueler@hotmail.com

Oscar Oliveira Santos

ORCID: https://orcid.org/0000-0002-9631-8480

Universidade Estadual de Maringá, Brasil

E-mail: oliveirasantos.oscardeoliveira@gmail.com

Jesuí Vergilio Visentainer

ORCID: https://orcid.org/0000-0003-3412-897X

Universidade Estadual de Maringá, Brasil

E-mail: jesuivv@gmail.com

Sandra Rojas Duailibi

ORCID: https://orcid.org/0000-0002-2973-7650

Universidade Estácio de Sá, Brasil

E-mail: srdconsultoria@gmail.com

\section{Resumo}

Segurança dos alimentos abrange conhecimentos e práticas referentes à saúde coletiva, com intuito de prevenir riscos associados à alimentação. Adjacente está o controle de qualidade, que associa fundamentos básicos para que o processo de produção de alimentos seja realizado evitando o surgimento de doenças transmitidas por alimentos (DTAs). DTAs são originadas pela ingestão de alimentos ou água contaminados, onde diversos fatores colaboram para emergência de doenças. Com isto, a Vigilância Sanitária apresenta como um dos papeis de fiscalização para liberação de licença sanitária. Sendo assim, o presente trabalho trata-se de uma revisão na literatura baseada nos programas de 
controle de qualidade em função da segurança dos alimentos com ênfase nas doenças transmitidas por alimentos. Para garantir a higiene dos produtos e a segurança dos alimentos, existem diversos programas, tais como: Boas Práticas de Fabricação (BPF) têm como objetivo evitar a contaminação dos produtos, desde a recepção das matérias-primas até o consumo. Programa de Autocontrole (PAC) principal ferramenta da agroindústria no controle dos processos de fabricação. Procedimento operacional padronizado (POP) consiste em descrever todas as operações para realizar determinado procedimento. Sistema de análise de perigos e pontos críticos de controle (APPCC) baseia-se na aplicação de princípios técnicos e científicos de prevenção, com finalidade de garantir inocuidade dos processos de produção, manipulação, transporte, distribuição e consumo dos alimentos. E método $5 \mathrm{~S}$ consiste em organizar o local de trabalho por meio de manutenção, limpeza, padronização e disciplina na realização do trabalho, com o mínimo de supervisão possível. Demonstra-se que todos programas caminham juntos.

Palavras-chave: Doenças transmitidas por alimentos; Boas práticas de fabricação na indústria de alimentos; Procedimento operacional padronizado; Vigilância sanitária.

\begin{abstract}
Food safety includes knowledge and practices related to collective health, in order to prevent risks associated with food. Adjacent is quality control, which combines basic foundations for the food production process to be carried out avoiding the emergence of foodborne diseases (DTAs). DTAs are caused by eating contaminated food or water, where several factors contribute to the emergence of diseases. With this, the Health Surveillance presents as one of the inspection roles for the release of the health license. Thus, the present work is a review of the literature based on quality control programs based on food safety with an emphasis on foodborne diseases. To guarantee product hygiene and food safety, there are several programs, such as: Good Manufacturing Practices (GMP) aim to avoid contamination of products, from the reception of raw materials to consumption. Self-Control Program (PAC), the main tool for agribusiness in the control of manufacturing processes. Standardized operating procedure (SOP) consists of describing all operations to perform a given procedure. Hazard analysis and critical control points (HACCP) system is based on the application of technical and scientific principles of prevention, with the purpose of guaranteeing the safety of the processes of production, handling, transportation, distribution and consumption of food. And the $5 \mathrm{~S}$ method consists of organizing the workplace through maintenance, cleaning, standardization and discipline in carrying out the work, with the least possible supervision. It is shown that all programs go together.
\end{abstract}

Keywords: Foodborne diseases; Good manufacturing practices in the food industry; Standardized operating procedure; Health surveilance.

\begin{abstract}
Resumen
La seguridad alimentaria incluye conocimientos y prácticas relacionados con la salud colectiva, con el fin de prevenir los riesgos asociados a los alimentos. Contiguo está el control de calidad, que combina los fundamentos básicos para que el proceso de producción de alimentos se lleve a cabo evitando la aparición de enfermedades de transmisión alimentaria (DTA). Los DTA son causados por comer alimentos o agua contaminados, donde varios factores contribuyen a la aparición de enfermedades. Con esto, la Vigilancia Sanitaria se presenta como uno de los roles de inspección para la liberación de la licencia sanitaria. Así, el presente trabajo es una revisión de la literatura basada en programas de control de calidad basados en la inocuidad alimentaria con énfasis en las enfermedades transmitidas por alimentos. Para garantizar la higiene del producto y la seguridad alimentaria, existen varios programas, tales como: Las Buenas Prácticas de Manufactura (BPM) tienen como objetivo evitar la contaminación de los productos, desde la recepción de las materias primas hasta el consumo. Programa de Autocontrol (PAC), la principal herramienta de la agroindustria en el control de los procesos de manufactura. El procedimiento operativo estandarizado (POE) consiste en describir todas las operaciones para realizar un procedimiento determinado. El sistema de análisis de peligros y puntos críticos de control (HACCP) se basa en la aplicación de principios técnicos y científicos de prevención, con el fin de garantizar la seguridad de los procesos de producción, manipulación, transporte, distribución y consumo de alimentos. Y el método 5S consiste en organizar el lugar de trabajo mediante el mantenimiento, la limpieza, la estandarización y la disciplina en la realización del trabajo, con la menor supervisión posible. Se muestra que todos los programas van juntos.
\end{abstract}

Palabras clave: Enfermedades transmitidas por alimentos; Buenas prácticas de fabricación en la industria alimentaria; Procedimiento operativo estandarizado; Vigilancia sanitaria.

\title{
1. Introdução
}

Segurança dos alimentos abrange conhecimentos e práticas referentes à saúde coletiva, com intuito de prevenir riscos associados à alimentação (Pandolfi, Moreira, \& Teixeira, 2020). Adjacente à isto está a/o gestão/controle de qualidade, que associa fundamentos básicos para que o processo de produção de alimentos seja realizado evitando o surgimento de doenças transmitidas por alimentos (DTAs) por alimentos DTAs (Pereira \& Zanardo, 2020). 
O aumento de DTAs apresenta-se de forma significativa pelo mundo inteiro, e, diversos fatores colaboram para a emergência destas doenças (de Andrade \& Sturion, 2015). DTAs são originadas pela ingestão de alimentos ou água contaminados (Brasil, 2017b). Porém, estudos relatam que a maioria dos casos não são notificados às autoridades sanitárias, pois muitos dos patógenos presentes nos alimentos causam sintomas leves (Oliveira et al., 2010).

Para garantir a higiene dos produtos e a segurança dos alimentos, deve-se ter como princípios a adoção de checklist, a organização de planilhas de controle e o treinamento de manipuladores, principalmente. Pois tais procedimentos deixam claro quais os pontos falhos no processo e propiciam a produção de um alimento de qualidade (Pereira \& Zanardo, 2020). Nesse contexto, existe a implementação do programa de Boas Práticas de Fabricação (BPF) em indústrias de alimentos.

Com isto, a vigilância sanitária apresenta como um dos papeis a fiscalização para liberação de licença sanitária, além de atendimento a denúncias, ações programadas, coleta de alimentos, investigação de surtos alimentares, análise de projetos arquitetônicos, análise de rotulagem de alimentos produzidos no município e atividades educativas (Brasil, 2017a).

Para Buzinaro e Gasparotto (2019) BPF's têm como objetivo primordial evitar a contaminação dos produtos, abrangendo desde a recepção das matérias-primas até o produto final. Sendo, na legislação brasileira, as BPF's obrigatórias para todos os estabelecimentos produtores e indústrias de alimentos.

Segurança dos alimentos é um tema atual e que deve ser sempre discutido e visado, uma vez que se refere à saúde da população, não apenas à qualidade dos produtos. Como foi visto através das possíveis doenças transmitidas por alimentos (DTAs), que são originadas pela ingestão de alimentos ou água contaminados e podem ocorrer devido às falhas no processo de gestão e controle de qualidade.

Sendo assim, o presente trabalho trata-se de uma revisão na literatura baseada nos programas de controle de qualidade em função da segurança alimentar com ênfase nas doenças transmitidas por alimentos.

\section{Metodologia}

O delineamento do estudo baseou-se em uma revisão bibliográfica com delineamento experimental, realizada em base de dados eletrônicos, buscando-se artigos científicos sobre o tema. Foi realizada busca em artigos científicos publicados em revistas, jornais periódicos, indexados nas bases de dados eletrônicos Science Direct, Google Acadêmico, Scientific Eletronic Library Online (Scielo), Agência Nacional de Vigilância Sanitária (ANVISA), Ministério da Saúde e Ministério da Agricultura, Pecuária e Abastecimento (MAPA).

\section{Resultados e Discussão}

No estudo realizado foram encontrados regulamentos normativos, resoluções e instruções de implantação de programa de qualidade regimentados no Brasil. Sendo assim, alguns deles apresentados a seguir:

\section{Segurança dos alimentos}

A segurança dos alimentos abrange conhecimentos e práticas referentes à saúde coletiva, com intuito de prevenir riscos associados à alimentação (Pandolfi, Moreira, \& Teixeira, 2020). Adjacente à segurança alimentar está a/o gestão/controle de qualidade, que associa fundamentos básicos para que o processo de produção de alimentos seja realizado evitando o surgimento de doenças transmitidas por alimentos (DTAs) (Pereira \& Zanardo, 2020). No setor alimentício, para a implantação de uma gestão da qualidade, é possível utilizar sistemas (métodos e procedimentos) já testados, além da literatura de fácil acesso (Sérvio et al., 2019). 


\section{Doenças transmitidas por alimentos (DTAs)}

As DTAs vêm aumentando mundialmente. Diversos fatores colaboram para a emergência destas doenças, entre eles: aumento da população, aumento de grupos vulneráveis, processo desordenado de urbanização e produção de alimentos em larga escala (de Andrade \& Sturion, 2015). DTAs, são doenças acometidas pela ingestão de alimentos e/ou água contaminados. Existem mais de 250 tipos de DTAs, sendo a maior parte infecções por bactérias e suas toxinas, vírus e parasitas (Brasil, 2017b). Agentes biológicos também são bastante conhecidos pelos prejuízos à segurança alimentar, contudo deve-se também atentar-se aos riscos físicos e químicos durante o processo de fabricação, manipulação, estocagem e fornecimento dos alimentos (Sebrae, 2018). Um surto alimentar de DTA é definida quando duas ou um grupo de pessoas apresentam a mesma enfermidade após o consumo de um mesmo alimento (ou água), e com a confirmação a partir das análises epidemiológicas (Vigilância Sanitária de Santa Catarina, 2017).

A Vigilância Sanitária (2017) menciona alguns dos fatores que influenciam na contaminação dos alimentos por agentes patogênicos: vítimas infectadas, práticas inadequadas de manipulação, higiene inadequada, recipientes tóxicos, aditivos acidentais ou intencionais. Existem fatores que também influenciam na proliferação dos agentes patogênicos oriundas da manipulação e armazenamento. E, por fim, existem fatores que influenciam na sobrevivência dos agentes patogênicos: aquecimento ou cocção insuficiente e reaquecimento insuficiente.

Devido ao consumo de alimentos fora do domicilio, a população preocupa-se com a qualidade dos alimentos consumidos (Pandolfi, Moreira \& Teixeira, 2020), a qual deixou de ser um diferencial competitivo e tornou-se uma condição permanente (Veronezi \& Caveião, 2016), porém, grande parte deste público, não possui informações necessárias para avaliar e exigir melhorias relacionadas à qualidade dos alimentos (Pandolfi, Moreira \& Teixeira, 2020).

As definições de qualidade sofreram mudanças significativas com o passar do tempo; sendo um simples conjunto de ações operacionais, centradas e localizadas em pequenas melhorias do processo produtivo, a um elemento fundamental do gerenciamento das organizações. E atualmente sendo fator crítico para a "sobrevivência" de empresas, produtos, processos e principalmente pessoas (David \& Guivant, 2020). Portanto, garantir a qualidade e segurança dos alimentos torna-se responsabilidade básica dos locais que fornecem alimentação, sendo este um alvo de constante preocupação dos órgãos fiscalizadores de saúde pública (Santos et al., 2014).

De acordo com Marchiori (2015), as empresas estão buscando implantar a gestão da qualidade visando uma melhoria contínua, exigência do mercado consumidor. Este sistema de gestão, controle de qualidade e treinamentos dos colaboradores são, atualmente, um diferencial na indústria alimentícia.

Já para Silva (2011), a qualidade e a segurança são as principais características dos produtos industrializados, pois esta postura é exigida pelos consumidores. E neste contexto a Agência Nacional de Vigilância Sanitária (ANVISA) regulamentou leis para controle sanitário de produção e comercialização de produtos na indústria alimentícia, dentre as quais estão: RDC n ${ }^{\circ}$ 275, de 21 de Outubro de 2002: que dispõe sobre as BPF e os POPs (Procedimentos Operacionais Padrões); Portaria SVS/MS n 326, de 30 de julho de 1997: que determina as Boas Práticas de Higiene Sanitária e BPF, segundo o Codex Alimentarius; Portaria MS n 1428, de 26 de Novembro de 1993: estabelece as diretrizes gerais de Boas Práticas de Produção e Prestação de Serviços na área de alimentos (Berti \& Santos, 2016). As indústrias alimentícias contam ainda com a certificação ISO 22000, criada em setembro de 2005, reconhecida internacionalmente, fundamentada nos princípios de segurança dos alimentos em toda cadeia da indústria alimentícia.

Ainda pensando na garantia da segurança dos alimentos e na prevenção de DTAs, devemos considerar que os

manipuladores apresentam papel importante em serviços de alimentação e, faze-los entender que tal importância é vital para a fluidez da produção com segurança (Pereira \& Zanardo, 2020). E pensando desta maneira, até mesmo a gestão de pessoas nas 
empresas alimentícias é importante, uma vez que, a qualificação de funcionários na área da segurança dos alimentos é essencial para manter a qualidade do processo geral (Barbosa et al., 2018).

DTAs são todas doenças causadas pela ingestão de perigos biológicos, químicos ou físicos presentes nos alimentos (Vigilância Sanitária de Santa Catarina, 2017). O alto risco de doenças transmitidas por alimentos (DTAs) na maioria dos consumidores possuem alto risco de doenças transmitidas por alimentos associadas a produtos alimenticios, porém há uma baixa probabilidade de infectar patógenos transmitidos por alimentos, entretanto, as agências regulatórias e de saúde pública podem desenvolver material de educação em segurança alimentar que contenha mais informações pessoais relevantes e enfatize a probabilidade relativamente alta de ocorrência de patógenos de origem alimentar. (Yu, Neal, \& Sirsat, 2018)

\section{Vigilância Sanitária aplicada em alimentos}

A regulamentação sanitária se realiza mediante a conjugação do conhecimento técnico multidisciplinar e do contexto político, implicando conciliação de interesses diversos e, com a expectativa de que o benefício à saúde coletiva seja o resultado principal. A regulação extrapola o mero ato fiscalizatório de caráter privativo do Estado, e o seu processo de formulação técnica e política tem como finalidade precípua a de ser, fundamentalmente, um dos veículos das políticas públicas dirigidas à prevenção de riscos e à promoção da saúde (Figueiredo, Recine, \& Monteiro, 2017).

O Centro de Vigilância Sanitária (2017) é de responsabilidade das três esferas governamentais e são compartilhadas entre os níveis de gestão, estruturado na forma de sistemas. Sendo que cada esfera tem uma responsabilidade, As equipes regionais e municipais de vigilância sanitária têm como responsabilidade, a execução das ações de controle sanitário na fabricação, distribuição e comércio de produtos alimentícios, dando segurança aos consumidores a partir da qualidade sanitária dos produtos. Essas ações são compartilhadas com outros órgãos, como o Ministério da Agricultura, Pecuária e Abastecimento (MAPA). De acordo com o Centro de Vigilância Sanitária (2017), possui missão de promover e proteger a saúde da população, com ações capazes de eliminar ou prevenir riscos à saúde decorrentes da alimentação, e para isso realiza, ações de monitoramento programado da qualidade sanitária de produtos e de estabelecimentos na área de alimentos.

Como principais atividades da vigilância sanitária podem-se citar: fiscalização para liberação de licença sanitária, atendimento a denúncias, ações programadas, coleta de alimentos, investigação de surtos alimentares, análise de projetos arquitetônicos, analise de rotulagem de alimentos produzidos no município e atividades educativas (Brasil, 2017a).

Marmentini e colaboradores (2015) afirmam que muitas praticas inadequadas ocorrem durante o processamento do alimento podendo facilitar a contaminação, a sobrevivência e a multiplicação de microrganismos causadores de DTAs. O conhecimento dos principais pontos de contaminação durante o processamento dos alimentos é essencial para garantir qualidade microbiológica e segurança ao consumidor.

\section{Formas de garantir a segurança dos alimentos nos serviços alimentícios}

A preocupação com a segurança dos alimentos servidos em estabelecimentos destinados a venda de refeições compreende o alimento pronto, e todas as etapas do processo, incluindo o recebimento da matéria prima, onde os produtos embalados devem ser observados com cuidado em relação à condição da embalagem, a qual pode dar a noção do alimento em seu interior (Barbosa et al., 2018). É sempre recomendado que os estabelecimentos adotem o uso de produtos sanitizantes nos vegetais, com o objetivo de higienizar todos os alimentos servidos sem tratamento térmico (Ferreira et al., 2013).

A qualidade das instalações e edificações dos estabelecimentos de serviços de alimentação tem alta relação com a precaução de contaminações do alimento, sendo que as instalações devem ser estruturadas de forma a evitar cruzamentos entre áreas limpas e contaminadas e devem ser mantidas em condições adequadas de higiene (Nunes, Adami \& Fassina, 2017). 
Ferreira et al. (2011) consideraram que aqueles estabelecimentos que possuem estruturas planejadas e que mantem uma rotina de higienização, são capazes de garantir controle eficiente de pragas, protegendo assim os alimentos.

De acordo com a Comissão do Código Sanitário da Junta da Organização das Nações Unidas para Alimentação e Agricultura (FAO, do inglês, Food and Agriculture Organization of the United Nations) e da Organização Mundial da Saúde (OMS), a higiene dos alimentos abrange medidas preventivas necessárias para preparação, manipulação, armazenamento, transporte e venda de alimentos, com intuito de garantir produtos inócuos, saudáveis e adequados ao consumo humano (Marins, 2014).

Nesse contexto, existe uma forma para a obtenção e garantia da qualidade e segurança nas industrias: a implementação do programa de Boas Práticas de Fabricação (BPF) qual têm como objetivo evitar a contaminação dos produtos, desde a recepção das matérias-primas até o consumo do produto. Programa de Autocontrole (PAC) principal ferramenta da agroindústria no controle dos processos de fabricação. Procedimento operacional padronizado (POP) consiste em descrever todas as operações para realizar determinado procedimento. Sistema de análise de perigos e pontos críticos de controle (APPCC) baseia-se na aplicação de princípios técnicos e científicos de prevenção, com finalidade de garantir inocuidade dos processos de produção, manipulação, transporte, distribuição e consumo dos alimentos. E método $5 \mathrm{~S}$ qual consiste em organizar o local de trabalho por meio de manutenção, limpeza, padronização e disciplina na realização do trabalho, com o mínimo de supervisão possível.

\section{Ferramenta 5S}

Entende-se que as BPF's devem fazer parte do sistema de gestão da segurança de alimentos, podendo ser implantadas previamente ou em conjunto com a APPCC, dependendo da necessidade e realidade de cada organização (Lourenço, 2020).

Para iniciar este processo, temos o Método dos 5S, que surgiu no Japão em meados do século XX e consiste em organizar o local de trabalho por meio de manutenção apenas do necessário, da limpeza, da padronização e da disciplina na realização do trabalho, com o mínimo de supervisão possível. Os $5 \mathrm{~S}$ são derivados de palavras japonesas que exprimem princípios fundamentais da organização (Lourenço, 2020), sendo:

1. SEIRI - Senso de utilização, arrumação, organização, seleção;

2. SEITON - Senso de ordenação, sistematização, classificação;

3. SEISO - Senso de limpeza, zelo;

4. SEIKETSU - Senso de asseio, higiene, saúde, integridade;

5. SHITSUKE - Senso de autodisciplina, educação, compromisso.

O 5S proporciona a mudança de comportamento das pessoas e do ambiente da empresa. Economia, organização, limpeza, higiene e disciplina tornam-se palavras comuns e praticadas por todos quando esta metodologia é aplicada. Estes fatores são fundamentais para elevar e garantir a produtividade (Lourenço, 2020).

\section{Boas práticas de fabricação $(B P F)$}

Para Veronezi (2015), BPF's são procedimentos que devem ser adotados pelas industrias com o objetivo de garantir a qualidade higiênico-sanitária de acordo com a legislação vigente. Já Buzinaro \& Gasparotto (2019) definem que BPF’s têm como objetivo primordial evitar a contaminação dos produtos, abrangendo desde a recepção das matérias-primas até o produto final. Sendo, na legislação brasileira, as BPF's obrigatórias para todos os estabelecimentos produtores e indústrias 
A Anvisa (2004) estabelece as BPF's como um conjunto de medidas adotadas pelas indústrias alimentícias no intuito de garantir qualidade sanitária e conformidade dos produtos com os regulamentos técnicos. A legislação sanitária federal regulamenta essas medidas em caráter geral, as quais se aplicam a todo tipo de indústria de alimentos, e são voltadas as indústrias que processam determinadas categorias de alimentos.

A elaboração e implantação do Manual de Boas Práticas são descritas por Zurlini et al. (2018) como fundamentais para que os alimentos sejam produzidos com qualidade, do ponto de vista da segurança alimentar e também nutricional.

Além disso, BPF's consistem em um dos sistemas mais reconhecidos e com boa resposta para a obtenção de alimento seguro, mantendo estreita relação com o consumidor, pois asseguram sua saúde, segurança e bem-estar. Promovem também educação e qualificação nos aspectos de higiene, desinfecção e disciplina operacional. Assim, a segurança de alimentos é garantida com esforços combinados de todos os envolvidos na cadeia produtiva (Veronezi, 2015).

\section{Pré-requisitos envolvidos nas Boas práticas de fabricação}

Segundo a resolução técnica (RDC 275/05), as BPF’s devem incluir: adequada edificação e higiene das instalações, apropriado tratamento de resíduos e efluentes, direcionamento para limpeza e manutenção, controle de qualidade da água, procedimento ideal para seleção e verificação de qualidade das matérias-primas e insumos, assim como certificação e manutenção de fornecedores, descrição das análises, níveis de contaminação e inspeções de matérias-primas e insumos e produtos acabados, corretas operações de recebimento, estocagem e transporte, treinamento para higiene pessoal, de equipamentos e de utensílios sanitários, aferição de instrumentos, formas de recolhimento de produtos (recall) e programa de manutenção preventiva, sendo estes inicialmente averiguados através de um checklist para verificação das não conformidades (Sandle, 2018).

\section{Implantação das Boas Práticas de Fabricação}

De acordo com Veronezi (2015), a implantação das BPF’s nos serviços de alimentação, além de ser uma exigência legal, eleva a qualidade dos produtos, protege a saúde publica, diminui gastos com internações hospitalares, da maior segurança e satisfação ao comensal. Para a implantação dessas BP, é necessário que se conheça o processo produtivo envolvido, bem como as limitações impostas pelo uso feito pelo cliente ou consumidor final, de forma a se ter uma visão sobre os perigos potenciais e os riscos de contaminação envolvidos. Com esses dados, é possível determinar o rigor e a profundidade das BPF's a serem implementadas. Para poder cobrar que as regras desse sistema sejam cumpridas pelos empregados, a empresa deve fornecer treinamento em manipulação de alimentos, incluindo programas de saúde e higiene pessoal a todos os novos colaboradores cujas atribuições estejam relacionadas com áreas de produção e controle de qualidade, sempre antes do início de suas atividades. O treinamento deve incluir também os colaboradores da área de manutenção e de outras áreas cuja atividade possa afetar a qualidade do produto. Periodicamente, e não excedendo o intervalo de um ano, os treinamentos devem ser reciclados e devidamente registrados. As indústrias de alimentos precisam descrever as operações realizadas em um manual, que consiste em um documento que inclua requisitos de manutenção preventiva e de higienização (instalações, dos equipamentos e dos utensílios), controle da agua de abastecimento, controle integrado de vetores e pragas urbanas, controle da higiene e saúde dos manipuladores e o controle e garantia de qualidade do produto final (Bertolino, 2010).

Para a implantação das BPF é necessário que se conheça o processo produtivo envolvido, bem como as limitações impostas pelo uso feito pelo cliente ou consumidor final, de forma a se ter uma visão sobre os perigos potenciais e os riscos de contaminação envolvidos. Com esses dados, será possível determinar o rigor e a profundidade das BPF's a serem implementadas (Senac, 2001). E para avaliar o nível de implantação das BPF's, é utilizada a lista de verificação (checklist) da RDC n 275 , de 21 de outubro de 2002 da ANVISA (2002). 
De forma geral, os itens que fazem parte do checklist das BPF's são: limpeza e conservação de instalações, qualidade da água, recebimento, estocagem e qualidade de matérias-primas, higiene pessoal, controle integrado de pragas, calibração de instrumentos e treinamento periódico para funcionários (Berthier, 2007).

Nesse contexto, em busca da garantia de saúde da população e qualidade dos alimentos, a ANVISA regulamentou leis para controle sanitário de produção e comercialização de produtos na indústria alimentícia, dentre as quais estão, a já citada $\mathrm{RDC} \mathrm{n}^{\circ}$ 275, de 21 de outubro de 2002: que dispõe sobre as BPF e os POP's (Procedimentos Operacionais Padrões); Portaria SVS/MS n ${ }^{\circ}$ 326, de 30 de julho de 1997: que determina as Boas Praticas de Higiene Sanitária e BPF, segundo o Codex Alimentarius; Portaria MS n 1428, de 26 de Novembro de 1993: estabelece as diretrizes gerais de Boas Práticas de Produção e Prestação de Serviços na área de alimentos (Berti \& Santos, 2016). As industrias alimentícias contam ainda com a certificação ISO 22000, criada em setembro de 2005, reconhecida internacionalmente, fundamentada nos princípios de segurança alimentar em toda cadeia da indústria alimentícia.

Outro ponto de extrema importância para o bom funcionamento de toda a cadeia de produção é a elaboração dos POP's, que consiste, segundo Sérvio, Souza e Pereira (2019), em descrever em detalhes todas as operações que são necessárias para realizar um determinado procedimento, ou seja, "um roteiro padronizado para realizar uma determinada atividade", fundamental dentro de qualquer processo funcional para garantir, mediante uma uniformização, os resultados desejados por cada tarefa realizada.

Para abranger todos estes requisitos, têm-se alguns passos que envolvem a elaboração de um Manual de BPF, sua implantação e a adequação das ferramentas que regem a qualidade, sendo os principais (Progeal, 2012):

1. Diagnóstico - Levantamento de todas as não conformidades encontradas no estabelecimento referente às Boas Práticas;

2. Plano de Ação - Orientações e sugestões para correção de todas as não conformidades identificadas nos diagnósticos;

3. Capacitação - Treinamento dos colaboradores em Boas Práticas no Manuseio correto dos Alimentos;

4. Manual de Boas Práticas (BP) - Elaboração do Manual de Boas Práticas na Manipulação dos Alimentos;

5. Manual de Procedimentos Operacionais Padronizados (POP) e Procedimentos Padronizados de Higiene Operacional (PPHO) - Elaboração do Manual dos Procedimentos;

6. Verificação Final (auditoria interna) - Levantamento geral da evolução da empresa com relação às Boas Práticas na Manipulação dos Alimentos.

As BPF's são obrigatórias pela legislação brasileira, no Brasil estas são estabelecidas por legislações federais, estaduais e municipais. No âmbito federal existem regulamentos gerais importantes publicados tanto pelo Ministério da Saúde quanto pelo Ministério da Agricultura Pecuária e Abastecimento (Lourenço, 2020). De inicio, podemos citar:

- Portaria MS n 1428/93: Precursora na regulamentação desse tema, dispõe, entre outras matérias, sobre as diretrizes gerais para o estabelecimento de Boas Práticas de Produção e Prestação de Serviços na área de alimentos.

- Portaria SVS/MS n 326/97: Baseada no "Código Internacional Recomendado de Práticas: Princípios Gerais de Higiene dos Alimentos", do Codex Alimentarius, estabelece requisitos gerais sobre as condições higiênico-sanitária e de BPF's para estabelecimentos produtores/industrializadores de alimentos.

- Portaria SVS/MS n 368/97: Apresenta o Regulamento Técnico sobre as condições higiênico-sanitárias e BPF's para Estabelecimentos Produtores de Alimentos. 
- Resolução - RDC n 275/05: Desenvolvida com o propósito de atualizar a legislação geral. Introduz o controle contínuo das BPF's e dos POP's, além de promover a harmonização das ações de inspeção sanitária por meio de instrumento genérico de verificação das Boas Práticas (BP).

Para a escrita do manual de BPF's da indústria é importante a utilização da RDC 275 e das portarias 326 e 368, as quais direcionam eficazmente esta realização. $O$ documento deve ser elaborado descrevendo as atividades realizadas na indústria de acordo com os requisitos exigidos pela legislação, contendo os POP's e respectivos Programas de Autocontrole (PAC), para a produção de alimentos seguros e de boa qualidade. Sendo este, uma reprodução fiel da realidade da empresa e estando sempre atualizado (Lourenço, 2020).

\section{Programa de Autocontrole (PAC)}

Principal ferramenta da agroindústria no controle dos processos de fabricação, visando à garantia da qualidade e inocuidade dos alimentos produzidos. Além disso, a implantação destes programas amplia a competitividade no mercado nacional e viabiliza a comercialização dos produtos no exterior, pois possibilita o atendimento as exigências dos países importadores (Lourenço, 2020). Estes programas são desenvolvidos, implantados e validados por estas empresas, visando à garantia de produção de alimentos seguros, conforme o Art. $12^{\circ}$ da Lei 8.078 de 11 de setembro de 1990, Código de Defesa do Consumidor (Brasil, 1990).

Os PACs conhecidos também como gestão da qualidade são definidos como o conjunto de boas práticas utilizadas nas diversas áreas funcionais da empresa, para obter-se, de forma eficaz e duradoura, a qualidade pretendida para um produto. E nesta busca por qualidade, é essencial que estas boas práticas, dentro das indústrias, sejam conduzidas de forma preventiva e não corretiva, preservando a segurança através do controle, evitando assim a transmissão de quaisquer doenças ao consumidor. Com isso, a preparação dos alimentos é necessária a busca pelo aperfeiçoamento contínuo de seus produtos e processos, a fim de continuarem nesse mercado cada vez mais exigente e globalizado (Ramos \& Vilela, 2016).

Os PACs estão ligados intrinsecamente a gestão de qualidade, asseguram a qualidade, os processos de fabricação são conhecidos, definidos, os envolvidos são capacitados e treinados. A elaboração e implementação dos PACs baseiam-se em primeiro lugar a concordância, apoio e inclusão de todos os colaboradores nos programas, qualificação de equipe, treinamentos, documentação escrita e revisada, além de atualizada de acordo com a fabricação na indústria de alimentos (Artilha-Mesquita et al., 2021).

\section{Procedimentos Operacionais Padrão (POP)}

De acordo com Buzinaro e Gasparotto (2019), procedimento operacional padrão consiste em descrever, com detalhes, todas as operações necessárias para realizar determinado procedimento, ou seja, um roteiro padronizado para realizar determinada atividade, sendo de grande importância dentro de qualquer processo funcional para garantir, mediante uniformização, os resultados desejados para cada tarefa realizada.

Para a ANVISA (2004), POP’s são procedimentos descritos de forma objetiva que definem as instruções para a realização de uma atividade na rotina da produção de alimentos, seja em sua elaboração, transporte ou armazenamento. Controle da potabilidade da água, manutenção preventiva e calibração de equipamentos, programa de recolhimento de alimentos, seleção de matérias-primas, ingredientes e embalagens, higienização das instalações, equipamentos, móveis e utensílios, manejo de resíduos e controle integrado de vetores e pragas urbanas são aspectos que requerem criação e manutenção de POP's. 
Para Buzinaro e Gasparotto (2019), POP's tem como objetivo a busca da uniformização do processo ou atividade, ou seja, fazer com que pessoas que executam a mesma tarefa possam realizá-las de forma invariável.

Ainda para Buzinaro e Gasparotto (2019), outro objetivo do POP é sustentar o processo em funcionamento, por meio da padronização e da minimização de ocorrências de desvios na execução de atividades; fazer com que açães tomadas para garantir a qualidade sejam padronizadas.

A elaboração de um POP deve seguir as seguintes etapas: objetivos, descrição, monitoramento, ação corretiva, registros e verificação, podendo existir uma variação de acordo com a legislação a ser seguida. Os POP's devem ser aprovados, datados e assinados pelo responsável pelo estabelecimento (Buzinaro e Gasparotto, 2019).

Os Procedimentos Padronizados de Higiene Operacional (PPHO) são recomendados pelo Food and Drug Administration (FDA) e até outubro de 2002 eram referência para o controle de procedimentos de higiene, até a criação da resolução RDC de ${ }^{\circ}$ 275, a qual criou e instituiu no Brasil os POP's, que vão além do controle da higiene, porém, não descaracterizam os PPHO, que continuam sendo utilizados. Entretanto o programa PPHO é mais utilizado nos estabelecimentos de leite e derivados, que funcionam sob regime de inspeção federal, como etapa preliminar de programas de qualidade como o APPCC (Lourenço, 2020).

\section{Sistema de análise de Perigos e Pontos críticos de Controle (APPCC)}

O sistema de análise de perigos e pontos críticos de controle (APPCC ou HACCP) baseia-se na aplicação de princípios técnicos e científicos de prevenção, com finalidade de garantir a inocuidade dos processos de produção, manipulação, transporte, distribuição e consumo dos alimentos, cobrindo todos os fatores que podem afetar sua segurança (Veronezi, 2015). No Brasil, a primeira legislação que remete ao APPCC surgiu em 1993, com procedimentos para o pescado. No mesmo ano, com a Portaria ${ }^{\circ}$ 1428, do Ministério da Saúde, foram estabelecidas normas para a aplicação do APPCC em todas as indústrias de alimentos do Brasil. Em 1998, a Portaria n 46 do MAPA (Ministério da Agricultura, Pecuária e Abastecimento) obrigou que fosse realizada a implantação gradativa do programa de garantia de qualidade APPCC em todas as indústrias de produtos de origem animal, cujo pré-requisito essencial são as Boas Praticas de Fabricação (Buzinaro \& Gasparotto, 2019). De acordo com Veronezi (2015), a referida Portaria ${ }^{\circ}$ 46, de 10 de fevereiro de 1998, define APPCC como "um sistema de análise que identifica perigos específicos e medidas preventivas para seu controle, objetivando a segurança do alimento".

O método APPCC baseia-se na avaliação completa do processo em estudo, identificando os perigos de contaminação de uma linha de produção, predominantemente os de origem microbiológica, mas também físicos e químicos, caracterizando matérias-primas e fatores intrínsecos e extrínsecos, como água, temperatura, $\mathrm{pH}$ e atmosfera que envolve o alimento, identificando, enfim, todo o histórico do produto (Marins, 2014).

\section{ISO 22.000}

ISO (a Organização Internacional para Padronização, do inglês International Organisation for Standardization) é uma federação mundial de órgãos nacionais (órgãos membros da ISO). ISO 22000 é baseada na estrutura de um sistema de gestão estruturado e incorporado nas atividades gerais de gestão da organização para estabelecer, implementar, monitorar e atualizar o mais eficaz sistema de segurança alimentar. Este padrão integra o sistema APPCC e as etapas de aplicação desenvolvidas pela Codex Alimentarius Commission. Pela aplicação de requisitos auditáveis, combina o Plano APPCC com demais programas de segurança de alimentos. É denominado sistemas de gestão da segurança alimentar (Iso, 2018).

Todos os perigos referidos a segurança de alimentos (biológicos, físicos, químicos, incluindo alérgenos) são esperados em relação ao tipo de produto, tipo de processo e instalações de processamento reais devem ser identificados e registrados 
(Codex Alimentarius Commission, 1996). A identificação deve ser baseada em (Codex Alimentarius Commission, 1999, WHO, 2008, Hoag, Porter, Uppala, \& Dyjack, 2007).

- As informações preliminares e os dados coletados de acordo com as características das matérias-primas, ingredientes, materiais de contato com o produto e produtos finais, uso pretendido, diagramas de fluxo, etapas do processo e medidas de controle

- Experiência (histórico de produto e produtor / processador)

- Informações externas, incluindo, na medida do possível, dados epidemiológicos e outros dados históricos (sistema de vigilância epidemiológica pelas autoridades de saúde / programa de monitoramento de contaminantes), e

- Informações da cadeia alimentar sobre os riscos à segurança alimentar que podem ser relevantes para a segurança dos produtos finais, produtos intermediários e alimentos no consumo (considerações ambientais).

Ao identificar os perigos, é importante considerar as etapas anteriores e posteriores à operação especificada, o equipamento do processo, utilidades / serviços e arredores e os elos anteriores e posteriores na cadeia alimentar. A análise de perigo, conforme detalhado na ISO 22000, exige que a organização avalie todas as medidas de controle de segurança de alimentos de forma científica. Assim que a análise de risco for concluída, isso pode resultar na adição de alguns novos PRPs ao sistema. Além disso, pode resultar na atualização de alguns PRPs para a categoria de PRP operacional. Portanto, é responsabilidade da organização documentar essas mudanças, aprová-las e se preparar para implementá-las de maneira adequada para garantir a segurança alimentar (Soman, \& Raman, 2016).

\section{Considerações Finais}

Segurança dos alimentos é um tema atual e que deve ser sempre discutido e visado, uma vez que se refere à saúde da população, não apenas à qualidade dos produtos. Como foi visto através das possíveis doenças transmitidas por alimentos (DTAs), que são originadas pela ingestão de alimentos ou água contaminados e podem ocorrer devido às falhas no processo de gestão e controle de qualidade. Por isto, com esta revisão da literatura demonstrando a existência dos principais programas de gestão de qualidade, que associam fundamentos básicos para o processo de produção de alimentos, podemos observar sua grande significância para as indústrias e consumidores finais. Com este trabalho, pode-se concluir uma abordagem de implementação de programas de controle de qualidade abrangendo a segurança dos alimentos por respectivos programas de qualidade como: 5S, BPF, PAC, POP, APPCC, ISO 22.000; quais todos programas, juntos, caminham e beneficiam tanto a indústria de alimentos, como a segurança de alimentos, desde o recebimento da matéria-prima até o consumidor. Além disso, demonstra-se que estes, caminham junto com a vigilância sanitária, colaborando à fiscalização para liberação, atendimento, ações programadas, coleta de alimentos, investigação de surtos alimentares, análise de projetos arquitetônicos, analise de rotulagem de alimentos produzidos e atividades educativas. Com isto demonstramos a interação existente entre vigilância sanitária e segurança dos alimentos de forma explicita e incontestável.

\section{Agradecimentos}

Os autores gostariam de agradecer à Coordenação de Aperfeiçoamento de Pessoal de Nível Superior (CAPES) e ao Conselho Nacional de Desenvolvimento Científico e Tecnológico (CNPq) pelo apoio financeiro e bolsas de estudos. Além disso, agradecemos ao grupo APLE-A e ao programa de Pós-Graduação em Ciência de Alimentos da UEM pelo apoio a pesquisa e estudos. 


\section{Referências}

Artilha-Mesquita, C. A. F., Stafussa, A. P., Paraiso, C. M., Rodrigues, L. M., da Silva, L. A., dos Santos, S. S., ... \& Madrona, G. S. (2021) Avaliação da Gestão da Qualidade e suas ferramentas: aplicabilidade em indústria de alimentos de origem animal. Research, Society and Development, 10(1), e20210111248-e20210111248.

Barbosa, Lorna Bandeira, Moreira, Marta da Rocha, Lustosa, Iramaia Bruno Silva, Brito, Fernando César Rodrigues, Sousa, Verlaine Suênia Silva de, \& Cabral, Lisidna de Almeida. (2018). Evaluation of good hygienic-sanitary practices in food trucks. Motricidade, 14(1), 226-231. http://www.scielo.mec.pt/scielo.php?script=sci_arttext\&pid=S1646-107X2018000100032\&lng=pt\&tlng=en.

Berthier, F. M. (2007). Ferramentas de gestão da segurança de alimentos: APPCC e ISO 22000 (uma revisão). Monografia de especialização. Universidade Federal de Brasília - UFB. http://bdm.unb.br/bitstream/10483/186/1/2007_FlorenceMarieBerthier.pdf.

Berti, R. C., \& Santos, D. C. (2016). Importância do controle de qualidade na indústria alimentícia: prováveis medidas para evitar contaminação por resíduos de limpeza em bebida UHT. Atas de Ciências da Saúde (ISSN 2448-3753), 4(1), 23-38. https://revistaseletronicas.fmu.br/index.php/ACIS/article/view/1084.

Bertolino, M. T. (2009). Gerenciamento da qualidade na indústria alimentícia: ênfase na segurança dos alimentos. Artmed Editora.

Brasil. (1990). Ministério da Justiça. Secretaria de Direito Econômico. Lei nº 8078, de 11 de setembro de 1990. Código de defesa do consumidor. Brasília, DF, 12 de novembro de 1990. http://www.planalto.gov.br/ccivil_03/leis/18078compilado.htm.

Brasil. (1993) Ministério da Saúde. Portaria n 1428, de 26 de novembro de 1993. Regulamento Técnico para Inspeção Sanitária de Alimentos. Diário Oficial da União, Poder Executivo, 02 de dezembro de 1993. http://bvsms.saude.gov.br/bvs/saudelegis/gm/1993/prt1428_26_11_1993.html.

Brasil. (1997) Ministério da Saúde. Secretaria de Vigilância Sanitária. Portaria SVS/MS 28 n 326, de 30 de julho de 1997. Condições Higiênicos Sanitárias e de Boas Práticas de Fabricação para Estabelecimentos Produtores/Industrializadores de Alimentos. https://bvsms.saude.gov.br/bvs/saudelegis/svs1/1997/prt0326_30_07_1997.html.

Brasil. (1997). Ministério da Agricultura, Pecuária e Abastecimento. Portaria SVS/MS n 368, de 04 de setembro de 1997. Regulamento Técnico sobre condições Higiênico Sanitárias e de Boas Práticas de Fabricação para Estabelecimentos Elaboradores/Industrializadores de Alimentos. https://www.gov.br/agricultura/pt-br/assuntos/inspecao/produtos-animal/empresario/Portaria_368.1997.pdf/view.

Brasil. (1998) Ministério da Saúde. Portaria n ${ }^{\circ}$ 46, de 10 de fevereiro de 1998. Manual Genérico de Procedimentos para APPCC em Indústrias de Produtos de Origem Animal. Diário Oficial da União, Poder Executivo, fev. https://www.agencia.cnptia.embrapa.br/Repositorio/PRT_046_10_02_199 8_MANUAL_GENERICO_DE_PROCEDIMENTOS_APPCCID-f4POhNOufV.pdf

Brasil. (2002). Resolução RDC no 275, de 21 de outubro de 2002. Regulamento Técnico de Procedimentos Operacionais Padronizados aplicados aos Estabelecimentos Produtores/Industrializadores de Alimentos e a Lista de Verificação das Boas Práticas de Fabricação em Estabelecimentos Produtores/Industrializadores de Alimentos. Diário Oficial da União, 2002. http://bvsms.saude.gov.br/bvs/saudelegis/anvisa/2002/ane xos/anexo_res0275_21_10_2002_rep.pdf.

Brasil. (2004) Agência Nacional de Vigilância Sanitária. Regularização de Empresas: Alimentos. 2004. http://portal.anvisa.gov.br/registros-eautorizacoes/alimentos/empresas/boas-praticas-de-fabricacao.

Brasil. (2004) Ministério da Saúde. Agência Nacional de Vigilância Sanitária. Resolução - RDC nº 216 , de 15 de setembro de 2004 . Dispõe sobre o Regulamento Técnico de Boas práticas para serviços de alimentação. Diário Oficial da República Federativa do Brasil, Brasília, DF, 2004. https://bvsms.saude.gov.br/bvs/saudelegis/anvisa/2004/res0216_15_09_2004.html.

Brasil. (2004) Ministério da Saúde. Portaria n 216, de 15 de setembro de 2004. Regulamento Técnico para Inspeção Sanitária de Alimentos. Diário Oficial da União, Poder Executivo, 15 de setembro de 2004.http://bvsms.saude.gov.br/bvs/saudelegis/anvisa/2004/res0216_15_09_2004.html.

Brasil. (2005) Ministério da Saúde. Agência Nacional de Vigilância Sanitária (ANVISA). Resolução RDC ${ }^{\circ} 275$, de 22 de setembro de 2005. Regulamento Técnico de Procedimentos Operacionais Padronizados aplicados aos Estabelecimentos Produtores/Industrializadores de Alimentos e a Lista de Verificação das Boas Práticas de Fabricação em Estabelecimentos Produtores/Industrializadores de Alimentos. http://bvsms.saude.gov.br/bvs/saudelegis/anvisa/2005/rdc0270_22_09_2005.html.

Brasil. (2017)a Ministério da Saúde. Atribuições da vigilância sanitária de alimentos. Portal da Saúde. http://www.saude.curitiba.pr.gov.br/vigilancia/sanitaria/separadoralimentos/atribuicoes-da-vigilancia-sanitaria-de-alimentos.html.

Brasil. (2017)b Ministério da Saúde. Doenças transmitidas por alimentos (DTA). http://portalsaude.saude.gov.br/index.php/oministerio/principal/secretarias/svs/doencas-transmitidas-por-alimentos- dta.

Buzinaro, D. V. C., \& Gasparotto, A. M. S. (2019). Como a implementação das boas práticas de fabricação (BPF) auxiliam a competitividade e a qualidade em uma indústria. Revista Interface Tecnológica, 16(2), 371-382. https://doi.org/10.31510/infa.v16i2.662.

Codex Alimentarius Commission. (1996). Principles for the establishment and application of microbiological criteria for foods. ALINORM 97/13A.

Codex Alimentarius Commission. (1999). Principles and guidelines for the conduct of microbiological risk assessment. $C A C / G L-30$.

Colenghi, V. M. (1997). O \& M e qualidade total: uma integração perfeita. Qualitymark.

David, M. L., \& Guivant, J. S. (2020). Os padrões de identidade e qualidade dos alimentos: uma análise de suas transformações no Brasil. Mediações: revista de ciências sociais. Londrina, PR. 25(1), 247-264. https://doi.org /10.5433/2176-6665.2020.1v25n1p247

de Andrade, M. L., \& Sturion, G. L. (2015). Segurança dos alimentos em serviços de alimentação do setor de turismo. Segurança Alimentar $e$ Nutricional, 22(1), 618-632. https://periodicos.sbu.unicamp.br/ojs/index.php/san/article/view/8641595. 
Ferreira, J.S., Cerqueira, E. S, Carvalho, J. S., Oliveira, L. C., Costa, W. L. R., \& Almeida, R. C. C (2013), Conhecimento, atitudes e práticas em segurança alimentar de manipuladores de alimentos em hospitais públicos de Salvador, Bahia. Revista Baiana de Saúde Pública, 37(1), 35-55. https://doi.org/10.22278/2318-2660.2013.v37.n0.a589.

Figueiredo, A. V. A., Recine, E., \& Monteiro, R. (2017). Regulação dos riscos dos alimentos: as tensões da Vigilância Sanitária no Brasil. Ciência \& Saúde Coletiva, 22, 2353-2366.

Furtini, L. \& Abreu, L. (2006) Utilização de APPCC na Indústria de Alimentos. Revista Ciência e Agrotecnologia. 30(2). https://doi.org/10.1590/S141370542006000200025 .

Hoag, M. A., Porter, C., Uppala, P. P., \& Dyjack, D. T. (2007). A risk-based food inspection program. Journal of environmental health, $69(7), 33-37$.

ISO, I. (2018). 22000: Food safety management systems-Requirements for any organization in the food chain. International Standard, 1-48.

Lourenço, B. M. S. G. (2020). Diagnóstico e avaliação dos sistemas de gestão da qualidade em uma empresa do setor alimentício viabilizando ações de melhorias (Bachelor's thesis, Universidade Tecnológica Federal do Paraná). http://repositorio.roca.utfpr.edu.br/jspui/handle/1/16320.

Marchiori, C. (2015). Diagnóstico e implantação de boas práticas de fabricação em uma indústria de conservas do Município de Francisco Beltrão, PR. http://repositorio.roca.utfpr.edu.br/jspui/handle/1/7185.

Marins, B. R. (2014). Segurança alimentar no contexto da vigilância sanitária: reflexões e práticas. https://www.arca.fiocruz.br/handle/icict/8649.

Marmentini, R.P.; Ronqui, L.; Alvarenga, V.A. A importância das boas práticas de manipulação para os estabelecimentos que manipulam alimentos. Revista Facimed, 40(8), 263-2015. https://docplayer.com.br/6172068-A-importancia-das-boas-praticas-de-manipulacao-para-os-estabelecimentos-que-manipulamalimentos.html.

Nunes, G. Q., Adami, F. S., \& Fassina, P. (2017). Boas práticas em serviços de alimentação escolar. Segurança Alimentar E Nutricional, 24(1), 26-32. https://doi.org/10.20396/san.v24i1.8648035.

Oliveira, A. B. A. D., Paula, C. M. D. D., Capalonga, R., Cardoso, M. R. D. I., \& Tondo, E. C. (2010). Doenças transmitidas por alimentos, principais agentes etiológicos e aspectos gerais: uma revisão. Revista HCPA. Porto Alegre. Vol. 30, n. 3 (Jul./set. 2010), 279-285. http://hdl.handle.net/10183/157808.

Pandolfi, I. A., Moreira, L. Q., \& Teixeira, E. M. B. (2020). Segurança alimentar e serviços de alimentação-revisão de literatura. Brazilian Journal of Development, 6(7), 42237-42246. https://doi.org/10.34117/bjdv6n7-002

Pereira, W. B. B., \& Zanardo, V. P. S. (2020). Gestão de boas práticas em uma cantina escolar. Vivências, 16(30), 193-200. https://doi.org/10.31512/vivencias.v16i30.152

Progeal. (2012) Programa de Gestão da Qualidade em Alimentos. Implantação e Implementação de BPF/GMP. http://www.progeal.com.br/site/pauloguimaraes-2/.

Ramos, G. V., \& Vilela, J. (2016). Implantação dos programas de autocontrole em indústrias de alimentos de origem animal. XII SEGeT: Simpósio de Excelência em Gestão e Tecnologia-Desenvolvimento de Competências Frente aos Desafios do Amanhã-Resende, RJ.

Sandle, T. (2018). Biocontamination Control for Pharmaceuticals and Healthcare. Academic Press.

Santos, C., Santos, E., Branco, V., Soares, C., \& Saraiva, A. (2014). Segurança alimentar em grupos de risco. Revista INFAD de Psicología. International Journal of Developmental and Educational Psychology, 6(1),337-342. https://doi.org/10.17060/ijodaep.2014.n1.v6.752

Sebrae (2018) Serviço de apoio às micro e pequenas empresas do amazonas-SEBRAE/AM. Boas Práticas para Manipuladores de Alimentos.https://datasebrae.com.br/wp-content/uploads/2019/11/Boas-pr\%C3\%A1ticas-para-manipuladores-de-alimentos.pdf.

Senac. (2001) Elementos de Apoio: Boas Práticas de Fabricação e sistema APPCC. (Qualidade e Segurança Alimentar). Projeto APPCC Mesa. Convênio CNC/CNI/SEBRAE/ANVISA. Rio de Janeiro, 278 p. https://pt.scribd.com/document/66004018/28747976-Elementos-de-Apoio-Boas-Praticas-e-SistemaAPPCC.

Sérvio, A. V., Souza, B. G, Pereira, R. C. D., Souza, C. G. G., \& Martins, T. C. F. (2019) Importância do Controle de Qualidade na Indústria de Alimentos. XII FAVE - Fórum Acadêmico da Faculdade Vértice - Univértix. https://fave.univertix.net/2019/11/04/importancia-do-controle-de-qualidade-na-industria-dealimentos/

Silva, E. M. D. (2011). Implantação das boas práticas de fabricação em uma agroindústria de produtos cárneos embutidos no município de São Jerônimo-RS. http://hdl.handle.net/10183/38139.

Sirtoli, D. B., \& Comarella, L. (2018). O papel da vigilância sanitária na prevenção das doenças transmitidas por alimentos (DTA). Revista Saúde e Desenvolvimento, 12(10), 197-209. https://www.uninter.com/revistasaude/index.php/saudeDesenvolvimento/article/view/878/507

Soman, R., \& Raman, M. (2016). HACCP system-hazard analysis and assessment, based on ISO 22000: 2005 methodology. Food Control, 69, 191-195. https://doi.org/10.1016/j.foodcont.2016.05.001

Veronezi, C. T., \& Caveião, C. (2016). A importância da implantação das boas práticas de fabricação na indústria de alimentos. Revista Saúde e Desenvolvimento, 8(4), 90-103.

Veronezi, C. T., \& Caveião, C. (2016). A importância da implantação das boas práticas de fabricação na indústria de alimentos. Revista Saúde $e$ Desenvolvimento, 8(4), 90-103. https://www.uninter.com/revistasaude/index.php/saudeDesenvolvimento/article/view/410. 
Research, Society and Development, v. 10, n. 1, e35810111687, 2021

(CC BY 4.0) | ISSN 2525-3409 | DOI: http://dx.doi.org/10.33448/rsd-v10i1.11687

Vigilância Sanitária Estado De Santa Catarina. (2017) Doença Transmitida por Alimento (DTA). Santa Catarina. http://www.vigilanciasanitaria.sc.gov.br/index.php/inspecao-de- produtos-e-servicos-de-saude/alimentos/91-area-de-atuacao/inspecao- de-produtos-e-servicosde-saude/alimentos/415-doenca-transmitida- por-alimento-dta.

WHO - World Health Organization. (2008). Exposure assessment of microbiological hazards in food: guidelines (Vol. 7). World Health Organization.

Yu, H., Neal, J. A., \& Sirsat, S. A. (2018). Consumers' food safety risk perceptions and willingness to pay for fresh-cut produce with lower risk of foodborne illness. Food Control, 86, 83-89

Zurlini, A. C., Lupino, C. S., Nery, J. S., \& Santos, M. C. H. (2018). Avaliação do controle higienicossanitário da produção de alimentos em unidades de alimentação e nutrição hospitalar. Hig. aliment, 51-55. https://docs.bvsalud.org/biblioref/2018/11/965436/284-285-set-out-2018-51-55.pdf 